\title{
Ley exponencial de la dinámica cardiaca caótica aplicada a 18 horas
}

\section{Exponential law of chaotic cardiac dynamics applied to 18 hours}

\author{
Javier-Oswaldo Rodríguez-Velásquez ${ }^{1}$; José-Fernando Giraldo-Cardona²; Freddy-Andrés Barrios-Arroyave²; \\ Signed-Esperanza Prieto-Bohórquez ${ }^{1}$; Sandra-Catalina Correa-Herrera' ${ }^{1}$, Maria-Yolanda Soracipa-Muñoz ${ }^{1}$
}

Forma de citar: Rodríguez-Velasquez JO, Giraldo-Cardona JF, Barrios-Arroyave FA, Prieto-Bohórquez SE, Correa-Herrera SC, Soracipa-Muñoz MY. Ley exponencial de la dinámica cardiaca caótica aplicada a 18 horas. Rev Univ Ind Santander Salud. 2019; 51(2): 147-154. doi: http://dx.doi.org/10.18273/revsal.v51n2-2019007 (c) (i)

\section{Resumen}

Introducción: La aplicación de una ley exponencial para los sistemas dinámicos caóticos cardiacos ha sido reducida a 18 horas para el análisis del Holter, cuantificando las dinámicas cardiacas normales y patológicas, así como la evolución entre estos estados. Metodología: Se analizaron 80 registros electrocardiográficos, 15 con dinámicas normales y 65 con diferentes patologías. Se construyó un atractor caótico para cada dinámica cardiaca a partir de la simulación de la secuencia de las frecuencias cardiacas durante 18 horas, posteriormente se halló la dimensión fractal de cada atractor y su ocupación espacial. Los parámetros diferenciadores de la ley caótica exponencial fueron aplicados diferenciando dinámicas cardiacas normales de aquellas patológicas, finalmente se calculó la sensibilidad, especificidad y coeficiente Kappa. Resultados: Las dinámicas normales presentaron espacios de ocupación por encima de 200 en la rejillla $\mathrm{Kp}$, y para la rejilla $\mathrm{Kg}$ por encima de 67. Para los casos de enfermedad aguda los valores en las rejillas $\mathrm{Kp} \mathrm{y} \mathrm{Kg}$ estuvieron por debajo de 73 y 22 respectivamente. Los valores de sensibilidad y especificidad fueron de $100 \%$ y el coeficiente Kappa fue de 1. Conclusión: La aplicación de la ley exponencial durante 18 horas mostro que fue posible caracterizar matemáticamente las dinámicas cardiacas, permitiendo reducir el tiempo de evaluación.

Palabras clave: Fractal; Diagnóstico; Electrocardiografía; Matemática; Enfermedad; Simulación.

\begin{abstract}
Introduction: The application of an exponential law for chaotic dynamic cardiac systems has been reduced to 18 hours for Holter analysis, quantifying normal and pathological cardiac dynamics, as well as the evolution between these states. Methodology: 80 electrocardiographic records were analyzed, 15 with normal dynamics and 65 with different pathologies. A chaotic attractor was constructed for each cardiac dynamic based on the simulation of the cardiac frequency sequence for 18 hours, after the fractal dimension of each attractor and its spatial occupation were found. The differentiating parameters of the chaotic exponential law were applied differentiating normal cardiac dynamics from those pathological, finally the sensitivity, specificity and Kappa coefficient were calculated. Results:

1. Grupo Insight. Bogotá D.C. Colombia.

2. Universidad de Manizales. Caldas, Colombia.

Correspondencia: Javier Rodríguez. Dirección: Cra. 79B N ํ 51-16 Sur. Int. 5. Bogotá D.C. Teléfono: +571 4522257. Correo electrónico: grupoinsight2025@gmail.com
\end{abstract}


The normal dynamics presented occupancy spaces above 200 in the Kp grid, and for the Kg grid above 67. In the cases of acute disease, the values in the Kp and $\mathrm{Kg}$ grids were below 73 and 22 respectively. The values of sensitivity and specificity were 100\% and the Kappa coefficient was 1. Conclusion: The application of the exponential law for 18 hours showed that it was possible to characterize mathematically the cardiac dynamics, allowing reducing the time of evaluation.

Keywords: Fractal; Diagnosis; Electrocardiography; Disease; Mathematics; Simulation.

\section{Introducción}

Predecir el comportamiento de un sistema a partir del cambio temporal de las variables dinámicas de éste, constituye el problema central de la teoría de los sistemas dinámicos ${ }^{1,2}$. Para el estudio de la dinámica de un sistema se utilizan representaciones gráficas en el espacio de fase, las cuales han sido denominadas atractores $^{3}$, generalmente se encuentran dos tipos de atractores; los predecibles y los impredecibles, los primeros corresponden a dinámicas como la del péndulo o el sistema solar, mientras que los impredecibles llevan a atractores caóticos que pueden estudiarse con la geometría fractal.

La geometría fractal fue desarrollada por Benoit Mandelbrot y estudia los objetos irregulares de la naturaleza ${ }^{4-6}$, mientras que los objetos geométricos regulares son estudiados por la geometría euclidiana. Varios tipos de fractales se han definido; los salvajes, los matemáticos y los estadísticos, para cada uno de estos tipos se asocia un cálculo especifico de la dimensión fractal. La dinámica cardiaca presenta un comportamiento caótico, por lo que su atractor corresponde a un fractal salvaje, que presenta superposición de sus partes, para calcular su dimensión fractal generalmente se utiliza el método de Box-counting?.

Se considera que las Enfermedades Cardiovasculares (ECV) son causadas por desórdenes cardiacos y de los vasos cardiacos, sus principales causas son el uso de tabaco, la inactividad física, dieta no saludable o el abuso de alcohol. De acuerdo a la Organización Mundial de la Salud (OMS $)^{8}$, las ECV son la principal causa de muertes a nivel mundial; y se estima que cerca de 23.6 millones de personas morirán debido a éstas en 2030, principalmente por enfermedad cardiaca y accidente cerebrovascular, continuando así en el primer lugar de las causas de mortalidad. Solamente en E.E.U.U. ${ }^{9}$, las ECV cobran anualmente, más vidas que el cáncer y Chronic Lower Respiratory Disease (CLRD) juntos.

Uno de los exámenes diagnóstico más utilizados para identificar alteraciones significativas del ritmo cardiaco de característica transitoria, de presentación súbita y condiciones asintomáticas es el Holter ${ }^{10}$, este examen es una prueba de electrocardiografía ambulatoria, de tipo no invasivo, que evalúa 2 o 3 canales electrocardiográficos durante un período de 24 a 48 horas. Dentro de la evaluación convencional de la dinámica cardiaca, el análisis de la Variabilidad de la Frecuencia Cardiaca (VFC) a partir de los cambios del intervalo RR en el tiempo, ha sido uno de los mayores campos de interés en la cardiología actual, debido a que su variabilidad, particularmente cuando se encuentra disminuida, se asocia a eventos como muerte súbita arrítmica $^{11}$, eventos cardiacos no arrítmicos como infarto agudo de miocardio ${ }^{12}$ y muerte por falla cardiaca ${ }^{13}$, además de eventos arrítmicos no fatales después de un infarto agudo de miocardio ${ }^{14}$.

Sin embargo, el análisis de la dinámica cardiaca y de la VFC desde teorías físicas y matemáticas ha controvertido muchos de los supuestos de la fisiología clásica e incluso la importancia de la VFC como predictor de eventos en la dinámica cardiaca, por ejemplo, a partir de la teoría de los sistemas dinámicos se ha desarrollado una reinterpretación del concepto de normalidad-enfermedad, en la que una dinámica cardiaca normal, lejos de comportarse de manera regular y cíclica como la fisiología clásica lo indica, se caracteriza por tener un comportamiento irregular e intermedio entre los dos extremos asociados a dinámicas patológicas que constituyen las dinámicas muy regulares o las extremadamente aleatorias ${ }^{15-19}$.

Con base en la metodología de evaluación hallada previamente ${ }^{20}$, la presente investigación busca analizar dinámicas cardiacas durante 18 horas con el fin de comprobar su capacidad de evaluación y su aplicabilidad clínica como ayuda diagnostica.

\section{Materiales y Métodos}

\section{Definiciones}

Atractor de frecuencias cardiacas: Mapa de retardo que se desarrolla a partir de graficar cada frecuencia cardiaca como función de la frecuencia cardiaca previa. 


\section{Dimensión Fractal de Box-Counting (D):}

$D=\frac{\log K p-\log K g}{\log 2_{K p}-\log 2_{K g}}=\log _{2} \frac{K p}{K g} \quad$ Ecuación 1

Donde Kp corresponde al número de cuadros ocupado por el objeto para la rejilla con partición $2_{K p}$ y Kg corresponde al número de cuadros ocupado por el objeto para la rejilla con partición $2_{\mathrm{Kg}}$.

Ley exponencial de la dinámica cardiaca caótica: De la ecuación 1 se obtiene una relación exponencial entre los espacios ocupados y la dimensión fractal (D):

$\mathrm{K}_{\mathrm{p}}=\mathrm{K}_{\mathrm{g}} 2^{\mathrm{D}}$

Ecuación 2

Población: Se tomaron en total 80 Holter o registros electrocardiográficos con una duración de mínimo 21 horas, de los cuales 15 correspondieron a casos diagnosticados por un cardiólogo experto como normales, y 65 casos correspondieron a casos diagnosticados con diferentes tipos de patologías pertenecientes a sujetos mayores de 21 años.

Procedimiento: De cada Holter o registros electrocardiográficos se tomaron los valores máximos y mínimos de las frecuencias cardiacas y el número total de latidos por hora, primero se tomó todo el registro y luego a partir de estos valores y de un programa previamente desarrollado (20) se generó una secuencia de las frecuencias cardiacas; estas fueron graficadas en el espacio de fases mediante un mapa de retardo (ver definiciones) en el cual se ubica una frecuencia cardíaca la una contra la siguiente en ambos ejes del mapa, construyendo de esta manera el atractor caótico de la dinámica cardiaca (Figura 1).

Luego, se calculó la dimensión fractal por medio del método de Box-counting (ecuación 1) superponiendo dos rejillas (Kp y Kg) para cuantificar los espacios ocupados por cada atractor y se estableció la evaluación física y matemática establecida previamente. De manera que, un Holter asociado a enfermedad será aquel que para su atractor caótico, se hallaron espacios de ocupación $\mathrm{Kp}$ inferiores a 73. Mientras que una dinámica normal se estableció cuando se encuentren espacios de ocupación $\mathrm{Kp}$ mayores de 200. El estado de evolución hacia la enfermedad se determina cuando se encuentren espacios de ocupación que se encuentren entre 73 y 200.

Posteriormente se desarrolló el mismo proceso, pero con sólo 18 horas para cada Holter, y una vez obtenidas las medidas matemáticas se compararon con las obtenidas con la totalidad del registro, en busca de similitudes o diferencias. Luego se buscaron concordancias o divergencias al comparar el diagnóstico físico-matemático aplicado a 18 horas y el diagnóstico convencional.

Análisis estadístico: Para el análisis estadístico que compara la metodología matemática con el Gold estándar; fue necesario utilizar una clasificación binaria, hallando Verdaderos Positivos (VP), Verdaderos Negativos (VN), Falsos Positivos (FP) y Falsos Negativos (FN) para calcular sensibilidad y especificidad, siendo cada uno de ellos:

$\mathrm{VP}=\operatorname{casos} \quad$ evaluados convencionalmente $\mathrm{y}$ matemáticamente como patológicos,

$\mathrm{VN}=\operatorname{casos} \quad$ evaluados convencionalmente $\mathrm{y}$ matemáticamente como normales,

$\mathrm{FP}=$ casos evaluados convencionalmente como normales, pero que presentaron valores matemáticos de enfermedad,

$\mathrm{FN}=$ casos evaluados convencionalmente como enfermos, pero que presentaron valores matemáticos de normalidad.

También se evaluó la concordancia entre el diagnóstico convencional y el físico-matemático por medio de la ecuación del coeficiente Kappa:

$K=\frac{C o-C a}{T o-C a}$

Donde, Co representa el número de concordancias observadas entre el diagnóstico matemático y el Gold estándar; To es el número total de casos; $\mathrm{C} a$ corresponde al número de concordancias atribuibles al azar que se calculan a través de la siguiente ecuación:

$C a=\left[\left(f_{1} x C_{1}\right) / T o\right]+\left[\left(f_{2} x C_{2}\right) / T o\right]$

Donde

$\mathrm{f}_{1}=$ número de Holter con valores matemáticos de normalidad,

$\mathrm{f}_{2}=$ número de casos evaluados matemáticamente como enfermedad,

$\mathrm{C}_{1}=$ número de casos diagnosticados como normales por el experto clínico,

$\mathrm{C}_{2}=$ número de casos diagnosticados desde el ámbito clínico convencional con alguna patología 


\section{Consideraciones éticas}

De acuerdo con la Resolución 8430 de 1993 del Ministerio de Salud colombiano, esta investigación presenta riesgo mínimo, pues los cálculos son realizados sobre bases de datos de reportes de exámenes que han sido prescritos previamente según protocolos establecidos convencionalmente. También se ha protegido el anonimato e integridad de los participantes, como lo reglamenta dicha resolución, así como la Declaración de Helsinki de la Asociación Médica Mundial.

\section{Resultados}

Al evaluar la totalidad del registro, se encontró que para la rejilla $\mathrm{Kp}$, los espacios de ocupación estuvieron entre 37 y 389, y para la rejilla $\mathrm{Kg}$ estuvieron entre 11 y 172, las dimensiones fractales estuvieron entre 0,7897 y 1,9234 .
Los Holter clasificados como normales presentaron valores de ocupación $\mathrm{Kp}$ mayores a 200 tanto para 21 como para 18 horas, lo que corresponde a una evaluación matemática de normalidad, y para la evaluación en 18 horas de los casos de Holter agudos se tuvieron valores de $\mathrm{Kp}$ menores a 73, siendo consistentes con lo encontrado previamente para 21 horas o más ${ }^{20}$.

El coeficiente Kappa fue de 1 y los valores de sensibilidad y especificidad fueron del $100 \%$, la evaluación matemática realizada por la metodología diferencia normalidad de enfermedad aguda, así mismo caracteriza numéricamente los casos que se encuentran evolucionando entre normalidad y enfermedad aguda. Al comparar las evaluaciones realizadas para 21 y 18 horas, se observa que las medidas son consistentes, evidenciando que la metodología es aplicable en 18 horas.

Tabla 1. Medidas de los espacios de ocupación de los atractores calculados con datos del Holter durante 21 horas y 18 horas.

\begin{tabular}{cccccccc} 
& & \multicolumn{3}{c}{$\mathbf{2 1}$ horas } & \multicolumn{3}{c}{$\mathbf{1 8}$ horas } \\
\cline { 3 - 8 } Holter No. & Indicaciones & Kp & $\mathbf{K g}$ & $\mathbf{D f}$ & $\mathbf{K p}$ & $\mathbf{K g}$ & $\mathbf{D f}$ \\
\hline 1 & Normal & 260 & 101 & 1,3642 & 263 & 99 & 1,4096 \\
2 & FA, fatiga & 138 & 79 & 0,8047 & 135 & 81 & 0,737 \\
3 & Chagas en estudio & 149 & 49 & 1,6045 & 149 & 50 & 1,5753 \\
4 & Normal & 314 & 139 & 1,1757 & 319 & 137 & 1,2194 \\
5 & Taquicardia de complejo amplio & 146 & 42 & 1,7975 & 148 & 44 & 1,75 \\
6 & Hiperventilación, episodio vasovagal & 91 & 24 & 1,9228 & 90 & 26 & 1,7914 \\
& Normal & 387 & 116 & 1,7382 & 392 & 118 & 1,7321 \\
7 & Normal & 324 & 170 & 0,9305 & 326 & 171 & 0,9309 \\
8 & Normal & 191 & 84 & 1,1851 & 194 & 82 & 1,2424 \\
9 & Insuficiencia respiratoria & 218 & 102 & 1,0958 & 215 & 102 & 1,0758 \\
10 & Normal & 80 & 35 & 1,1926 & 81 & 35 & 1,2106 \\
11 & Taquicardia ventricular idiopática & 207 & 70 & 1,5642 & 206 & 71 & 1,5368 \\
12 & Arritmia en estudio & 165 & 65 & 1,344 & 164 & 64 & 1,3576 \\
13 & Fibrilación auricular & 38 & 11 & 1,7885 & 36 & 11 & 1,71 \\
14 & Infarto del miocardio sin elevación ST, SCA & 73 & 22 & 1,7304 & 71 & 20 & 1,8278 \\
15 & Normal & 299 & 79 & 1,9202 & 295 & 80 & 1,8826 \\
16 & Normal & 293 & 93 & 1,6556 & 291 & 91 & 1,6771 \\
17 & Normal & 324 & 88 & 1,8804 & 325 & 88 & 1,8849 \\
18 & Normal & 216 & 67 & 1,6888 & 216 & 66 & 1,7105 \\
19 & Normal & 254 & 72 & 1,8188 & 251 & 77 & 1,704 \\
20 & Miocarditis & 190 & 55 & 1,7885 & 192 & 56 & 1,7776 \\
21 & Insuficiencia cardíaca congestiva & 177 & 62 & 1,5134 & 181 & 62 & 1,5456 \\
\hline
\end{tabular}

Kp: rejilla con cuadros pequeños.

$\mathrm{Kg}$ : rejilla con cuadros grandes.

Df: dimensión fractal. 


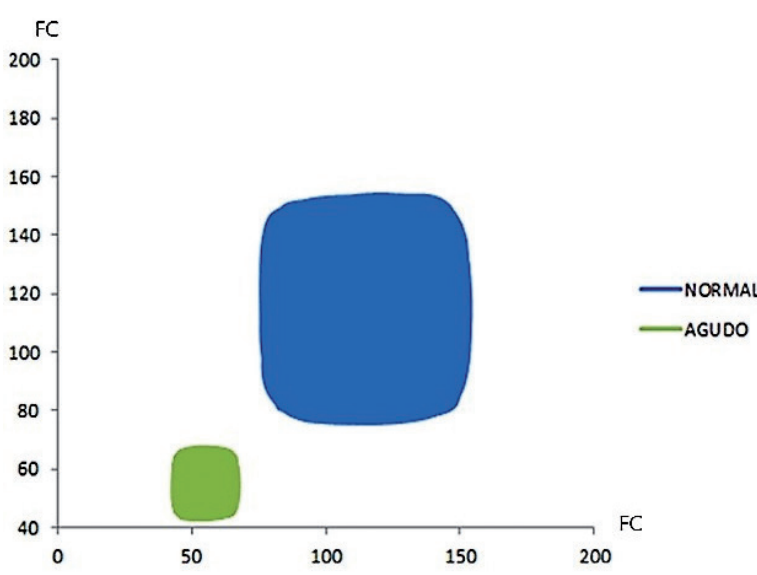

Figura 1. Atractores caóticos de la dinámica cardiaca. Ejemplos de dinámicas normal y aguda, correspondiente a los Holter 19 y 13, respectivamente (Tabla 1)

FC: frecuencia cardíaca

\section{Discusión}

La ley de la dinámica caótica cardiaca exponencial permitió deducir la totalidad de posibles atractores cardiacos; la dinámica cardiaca presenta un comportamiento caótico, y la representación gráfica de dicha dinámica presenta una forma irregular que puede ser caracterizada con la geometría fractal, esta geometría ha sido aplicada en diferentes ámbitos de la medicina ${ }^{21-25}$ buscando diferenciar normalidad de enfermedad, sin embargo, las dimensiones fractales aisladas han mostrado no ser concluyentes para lograr esta diferenciación ${ }^{26,27}$, por esto se han desarrollado diferentes conceptos para lograr dicha diferenciación ${ }^{28,29}$. De igual forma, en la dinámica cardiaca, las dimensiones fractales aisladas han mostrado ser insuficientes para diferenciar normalidad y enfermedad ${ }^{20,30,31}$, siendo los espacios de ocupación del atractor, lo que permite establecer un diagnóstico para cada caso particular, independientemente de metodologías estadísticas y epidemiológicas que describen comportamientos poblacionales.

A partir de métodos lineales, la teoría del caos y el análisis fractal se han desarrollado nuevas metodologías para el estudio de las dinámicas fisiológicas ${ }^{32-38}$, con esto se han logrado contribuciones importantes, entre ellas, una nueva concepción de normalidad enfermedad ${ }^{15}$, así como mejores predictores de mortalidad, y nuevos índices para cuantificar la complejidad de las dinámicas estudiadas, sin embargo es necesario realizar más estudios para establecer su aplicabilidad clínica ${ }^{39,40}$.

El máximo nivel de concordancia (coeficiente Kappa) entre el diagnóstico físico matemático y el Gold- estándar fue obtenido, de igual forma la sensibilidad y especificidad fue del $100 \%$ mostrando así que la metodología fue capaz de diferenciar objetiva y cuantitativamente normalidad de enfermedad, permitiendo reducir el tiempo de evaluación de la dinámica cardiaca, lo que podría ser de ayuda en la rapidez con la que el paciente sea evaluado, contribuyendo a mejorar la atención de éste.

A partir de lo anterior, es posible concluir que la metodología caótica matemática puede discriminar el estado de la dinámica cardíaca, diferenciando la normalidad de la enfermedad crónica de la aguda mediante la cuantificación los espacios de ocupación de los atractores y que ésta soporta una reducción del tiempo de evaluación a 18 horas manteniendo su misma capacidad diagnóstica. La reducción del tiempo de evaluación ha sido evaluada anteriormente en otros estudio a 16 horas $^{41}$, cuyos valores son comparados con los obtenidos en esta investigación (Tabla 2), confirmando que los valores de la dimensión fractal no permiten obtener diferencias entre ningún estado, mientras que los espacios de ocupación de la rejilla $\mathrm{Kp}$ si lo permiten en el $100 \%$ de los casos, con lo cual se ratifica la capacidad diagnóstica de la metodología implementada.

Tabla 2. Comparación de los resultados obtenidos de la dimensión fractal y la rejilla Kp en 16 y 18 horas.

\begin{tabular}{llcccc} 
& & \multicolumn{2}{c}{$\mathbf{1 6}$ horas } & \multicolumn{2}{c}{$\mathbf{1 8}$ horas } \\
\cline { 3 - 6 } & & Kp & Df & Kp & Df \\
\hline \multirow{2}{*}{ Totalidad } & Mín. & 46 & 0.81 & 37 & 0.71 \\
& Máx. & 350 & 1.98 & 389 & 1.89 \\
\multirow{2}{*}{ Normalidad } & Mín. & & 0.81 & & 0.93 \\
& Máx. & $\geq 200$ & 1.89 & $\geq 200$ & 1.88 \\
& Mín. & 74 & 0.82 & 74 & 0.71 \\
\multirow{4}{*}{ Agudización } & Máx. & 199 & 1.98 & 199 & 1.79 \\
& Mín. & & 1.25 & & 1.71 \\
& Máx. & & 1.89 & $\leq 73$ & 1.82 \\
\hline
\end{tabular}

Esta metodología, tanto a 18 como a 16 horas, podría indicarse en la práctica clínica cotidiana para realizar el seguimiento de pacientes en las Unidades de Cuidados Intensivos (UCI) en el contexto de enfermedades agudas como las patologías isquémicas cardiacas o en el postoperatorio, como en la cirugía cardiovascular, ya que mostraría la evolución de la dinámica en el tiempo y le indicaría a los clínicos si las intervenciones realizadas han tenido éxito o si existe algún deterioro, sin tener que lidiar con factores subjetivos de interpretación al ser una medida netamente cuantitativa, siendo más útil con mayores reducciones de horas de evaluación. 
También se indicaría en los pacientes ambulatorios a quienes se haya indicado un estudio Holter por cualquier razón ya que resulta útil evaluar la evolución de la dinámica cardíaca en la consulta externa para realizar el seguimiento de pacientes con enfermedades crónicas como las arritmias cardíacas. Sin embargo, deben realizarse estudios con distintos escenarios clínicos y distintas horas de registro para confirmar los hallazgos experimentales. Su mayor utilidad resultará de su automatización para proveer información útil y objetiva a los clínicos.

En otros ámbitos de la medicina se ha logrado llegar a generalizaciones que permiten establecer predicciones de un fenómeno específico como en el campo de la la epidemiología ${ }^{42}$, la morfometría ${ }^{28,29}$ y la infectología ${ }^{43}$. En el campo de la biología molecular y la inmunología, se desarrolló una teoría que permite la predicción del fenómeno de unión de péptidos al HLA clase 2 desde la probabilidad y la entropía ${ }^{44}$, y de igual forma en el campo de la cardiología ${ }^{45}$. También se han desarrollado predicciones en la $\mathrm{UCI}^{46}$ de mortalidad mediante la teoría de conjuntos aplicada a atractores de variables hemodinámicas.

\section{Agradecimientos}

Agradecemos a la Universidad de Manizales, por la financiación del presente proyecto. De igual forma al Centro de Investigaciones de la Clínica del Country por apoyo a nuestras investigaciones; a los Doctores Tito Tulio Roa, Director de Educación Médica, Jorge Ospina, Director Médico, Alfonso Correa, Director del Centro de Investigaciones, y a las Doctoras Adriana Lizbeth, epidemióloga, Silvia Ortiz, Enfermera Jefe, y Sandra Rodríguez enfermera, del Centro de Investigaciones.

\section{Referencias}

1. Devaney R. A first course in chaotic dynamical systems theory and experiments. Reading Mass. Addison-Wesley 1992. doi: 10.1063/1.4823195.

2. Peitgen H, Jürgens H, Saupe D. Strange attractors, the locus of chaos. En: Chaos and Fractals: New Frontiers of Science. Springer-Verlag. NY. 1992; pp. 655-768. doi: 10.1007/b97624

3. Calabrese JL. Ampliando las fronteras del reduccionismo. Deducción y sistemas no lineales. Psicoanálisis ApdeBA. 1999; 21(3): 431-453.

4. Mandelbrot B. Cambios de escala y leyes potenciales sin geometría. En: The fractal geometry of nature. Freeman Ed. San Francisco, 1972: pp. 477-487. doi: $10.1119 / 1.13295$
5. Mandelbrot B. Árboles jerárquicos o de clasificación, y la dimensión. En: Los Objetos Fractales. Tusquets Eds S.A. Barcelona. 2000; 161-166.

6. Mandelbrot B. How Long Is the Coast of Britain? statistical self-similarity and fractional dimension. Science. 1967; 156: 636-638. doi: 10.1126/ science.156.3775.636.

7. Peitgen H, Jürgens H, Saupe D. The Box-Counting Dimension En: Chaos and Chaos and Fractals: New Frontiers of Science. Springer-Verlag. N.Y. 1992. DOI: $10.1007 / \mathrm{b} 97624$

8. World Health Organization. Noncommunicable diseases. World Health Organization; 2018.

9. Mozaffarian D, Benjamin E, Go AS, Arnett DK, Blaha MJ, Cushman M, et al. Heart Disease and Stroke Statistics-2015 Update. A report from the American heart association. Circulation. 2015; 131: e29-e322. doi: 10.1161/CIR.0000000000000152.

10. Steinberg JS, Varma N, Cygankiewicz I, Aziz P, Balsam P, Baranchuk A, et al. 2017 ISHNEHRS expert consensus statement on ambulatory ECG and external cardiacmonitoring/telemetry. Heart Rhythm. 2017; 14: e55-e96. doi: 10.1016/j. hrthm.2017.03.038.

11. Barron H, Viskin S. Autonomic markers and prediction of cardiac death after myocardial infarction. Lancet. 1998; 351: 461-462. doi: 10.1016/S0140-6736(05)78676-1.

12. Hilldebrand S, Gast KB, de Mutsert R, Swenne CA, Jukema JW, Middeldorp, et al. Heart rate variability and first cardiovascular event in populations without known cardiovascular disease: meta-analysis and dose-response meta-regression. EP Europace. 2013; 15(5): 742-749. doi: 10.1093/europace/eus341.

13. Lui G, Wang L, Wang Q, Zhou G, Wang Y, Jiang Q. A New approach to detect congestive heart failure using short-term heart rate variability measures. PLoS One. 2014; 9(4): e93399. doi: 10.1371/ journal.pone.0093399.

14. Wolf M, Varigos G, Hunt D, Sluman J. Sinus arrhythmia in acute myocardial infarction. Med J Aus. 1978; 2: 52-53. doi: 10.5694/j.13265377.1925.tb11693.x.

15. Goldberger A, Amaral L, Hausdorff JM, Ivanov P, Peng Ch, Stanley HE. Fractal dynamics in physiology: alterations with disease and aging. PNAS. 2002; 99: 2466-2472. doi: 10.1073/ pnas.012579499.

16. Higgins JP. Nonlinear systems in medicine. Yale J Biol Med. 2002; 75: 247-260.

17. Costa M, Goldberger AL, Peng CK. Multiscale Entropy Analysis of Complex Physiologic Time Series. Phys Rev Lett. 2002; 89(6): 0681021- 
0681024. doi: 10.1103/PhysRevLett.89.068102.

18. Wu GQ, Arzeno NM, Shen LL, Tang DK, Zheng DA, Zhao NQ, et al. Chaotic signatures of heart rate variability and its power spectrum in health, aging and heart failure. PLoS ONE. 2009; e4323. doi: 10.1371/journal.pone.0004323.

19. Braun C, Kowallik P, Freking A, Hadeler D, Kniffki K, Meesmann M. Demonstration of nonlinear components in heart rate variability of healthy persons. Am J Physiol. 1998; 275: H1577-H1584. doi: 10.1152/ajpheart.1998.275.5.H1577.

20. Rodríguez J. Mathematical law of chaotic cardiac dynamic: Predictions of clinic application. J Med Med Sci. 2011; 2(8): 1050-1059.

21. Huikuri HV, Mäkikallio TH, Peng Ch, Goldberger AL, Hintze U, Moller M. Fractal correlation properties of R-R interval dynamics and mortality in patients with depressed left ventricular function after an acute myocardial infartion. Circulation. 2000; 101: 47-53. doi: 10.1161/01.CIR.101.1.47.

22. Barwad A, Dey P. Multifractal spectrum differentiation ofwell-differentiat ed adenocarcinoma from complex atypical hyperplasia of the uterus. Anal Quant Cytol Histol. 2012; 34(2): 105-108.

23. Moreno PA, Vélez PE, Martínez E, Garreta LE, Díaz N, Amador S, et al. The human genome: a multifractal analysis. BMC Genomics. 2011; 12: 506. doi: 10.1186/1471-2164-12-506.

24. Bikou O, Delides A, Drougou A, Nonni A, Patsouris E, Pavlakis K. Fractal dimension as a diagnostic tool of complex endometrial hyperplasia and welldifferentiated endometrioid carcinoma. In Vivo. 2016; 30(5): 681-690.

25. Zatloukal Z. Granulometry and fractal dimensions. Ceska Slov Farm. 2003; 52(5): 244-247.

26. Saidov T, Heneweer C, Kuenen M, von Broich, Wijkstra H, Rosette J, et al. Fractal dimension of tumor microvasculature by DCE-US: preliminary study in mice. Ultrasound Med Biol. 2016; 42(12): 2852-2863. doi: 10.1016/j. ultrasmedbio.2016.08.001.

27. Verma G, Luciani ML, Palombo A, Metaxa L, Panzironi G, Pediconi F, et al. Microcalcification morphological descriptors and parenchyma fractal dimension hierarchically interact in breast cancer: A diagnostic perspective. Comput Biol Med. 2018; 93: 16. doi: 10.1016/j.compbiomed.2017.12.004.

28. Rodríguez J, Prieto S, Correa C, Bernal P, Puerta $\mathrm{G}$, Vitery $\mathrm{S}$, et al. Theoretical generalization of normal and sick coronary arteries with fractal dimensions and the arterial intrinsic mathematical harmony. BMC Medical Physics. 2010; 10:1-6. doi:
10.1186/1756-6649-10-1.

29. Rodríguez J, Prieto S, Correa C, Posso H, Bernal P, Puerta G, et al. Generalización fractal de células preneoplásicas y cancerígenas del epitelio escamoso cervical. Una nueva metodología de aplicación clínica. Rev Fac Med. 2010; 18(2):173-181.

30. Rodríguez J, Correa C, Melo M, Domínguez D, Prieto S, Cardona DM, et al. Chaotic cardiac law: Developing predictions of clinical application. J Med Med Sci. 2013;4(2): 79-84.

31. Rodríguez J, Narváez R, Prieto S, Correa C, Bernal P, Aguirre G, Soracipa Y, Mora J. The mathematical law of chaotic dynamics applied to cardiac arrhythmias. J. Med. Med. Sci. 2013; 4(7): 291-300.

32. Gao J, Hu J, Liu F, Cao Y. Multiscale entropy analysis of biological signals: a fundamental biscaling law. Front Comput Neurosci. 2015; 9:64. doi: 10.3389/fncom.2015.00064.

33. Nogueira ML, Garner DM, Osório E, de Abreu LC, Valenti VE. Globally chaotic analysis of Heart Rate Variability during acute auditory stimulus by heavy metal music. Medical Express. 2015;2(5): 1-7. doi: 10.5935/MedicalExpress.2015.05.04.

34. Krogh T, Christini DJ. Nonlinear dynamics in cardiology. Annu Rev Biomed Eng. 2012; 14: 179203. doi: 10.1146/annurev-bioeng-071811-150106.

35. Huikuri HV, Mäkikallio TH, Peng Ch, Goldberger AL, Hintze U, Moller M. Fractal correlation properties of R-R interval dynamics and mortality in patients with depressed left ventricular function after an acute myocardial infartion. Circulation. 2000; 101: 47-53.

36. Porta A, Guzzetti S, Montano N, Furlan R, Pagani M, Malliani A. et al. Entropy, entropy rate and pattern classification as tools to typify complexity in short heart period variability series. IEEE Trans. Biomed Eng. 2001; 48: 1282-1291.

37. Guzzetti S, Borroni E, Garbelli PE, Ceriani E, Della $\mathrm{P}$, Montano N, et al. Symbolic dynamics of heart rate variability: a probe to investigate cardiac autonomic modulation. Circulation. 2005; 112: 465-470. doi: 10.1161/CIRCULATIONAHA.104.518449.

38. Maestri R, Pinna GD, Accardo A, Allegrini P, Balocchi R, D'Addio G, et al. Nonlinear indices of heart rate variability in chronic heart failure patients: redundancy and comparative clinical value. J Cardiovasc Electrophysiol. 2017; 18: 425433. doi: 10.1111/j.1540-8167.2007.00728.x.

39. Voss A, Schulz S, Schroeder R, Baumert M, Caminal P. Methods derived from nonlinear dynamics for analysing heart rate variability. Philos Trans A Math Phys Eng Sci. 2009; 367: 277-96. doi: 10.1098/ rsta.2008.0232. 
40. Perkiömäki J, Mäkikallio TH, Huikuri HV. Fractal and complexity measures of Heart Rate variability. Clin Exp Hypertens. 2005; 2:149-158. doi: 10.1081/ CEH-48742.

41. Rodríguez J, Correa C, Prieto S, Valencia LE, Barrios FA. Evaluación de la dinámica cardiaca a partir de la ley caótica exponencial: reducción a 16 horas. Arch Medicina. 2017; 13(2):3. doi: 10.3823/1343.

42. Rodríguez J. Método para la predicción de la dinámica temporal de la malaria en los municipios de Colombia. Rev Panam Salud Pública 2010; 27(3):211-218.

43. Rodríguez J, Prieto S, Correa C, Melo M, Dominguez D, Olarte N, et al. Prediction of CD4+ Cells counts in HIV/AIDS patients based on sets and probability Theories. Current HIV Research. 2018;16(6). doi: 10.2174/1570162X17666190306125819.

44. Rodríguez J. Teoría de unión al HLA clase II teorías de Probabilidad Combinatoria y Entropía aplicadas a secuencias peptídicas. Inmunología 2008; 27(4): 151-66. doi: 10.1016/S0213-9626(08)70064-7.

45. Rodríguez J. Entropía proporcional de los sistemas dinámicos cardiacos: predicciones físicas y matemáticas de la dinámica cardiaca de aplicación clínica. Rev Colomb Cardiol 2010; 17: 115-129. doi: 10.1016/S0120-5633(10)70229-1.

46. Rodríguez J. Dynamical systems applied to dynamic variables of patients from the intensive care unit (ICU): Physical and mathematical mortality predictions on ICU. J Med Med Sci. 2015; 6(8): 209-220. 